\title{
De novo formation of a carotid web: case report
}

\author{
Giovanni G. Vercelli, MD, ${ }^{1,4}$ Norbert G. Campeau, MD, ${ }^{2}$ Thanila A. Macedo, MD, ${ }^{2}$ \\ Elliot T. Dawson, MD, ${ }^{3}$ and Giuseppe Lanzino, MD ${ }^{1,2}$
}

\begin{abstract}
Departments of ${ }^{1}$ Neurologic Surgery, ${ }^{2}$ Radiology, and ${ }^{3}$ Neurology, Mayo School of Medicine, Mayo Clinic, Rochester, Minnesota; and ${ }^{4}$ Neurosurgical Unit, Department of Neuroscience, University of Turin, Italy
\end{abstract}

\begin{abstract}
A carotid web is a shelf-like intraluminal filling defect typically arising from the posterolateral wall of the proximal internal carotid artery. It is recognized as a possible cause of ischemic stroke in young adults. However, its etiopathogenesis is controversial and remains to be fully elucidated. The authors report de novo formation of a carotid web from an intimal dissection documented on serial imaging studies. The findings in this case suggest that a focal intimal dissection could be the underlying cause of a carotid web. Lower shear stress at the posterolateral wall of the proximal internal carotid artery is hypothesized to be a predisposing factor and explains the predilection of a carotid web for this specific location. https://thejns.org/doi/abs/10.3171/2018.7.JNS181579
\end{abstract}

KEYWORDS carotid web; de novo formation; pathogenesis; carotid diaphragm; vascular disorders

$\Lambda$ CAROTID web is defined as a shelf-like intraluminal filling defect arising from the posterolateral wall of the proximal internal carotid artery (ICA). The proximity of the luminal defect to the carotid bulb creates thrombogenic flow parameters, ${ }^{7,8,14}$ which in turn lead to thrombus formation and secondary emboli. ${ }^{2}$ A carotid web has long been considered a possible cause of ischemic stroke in young adults, and recent studies have confirmed this association. ${ }^{3,11}$ The pathophysiology underlying the formation of a carotid web is largely unknown and controversial.

We illustrate de novo formation of a carotid web on serial imaging studies and document an intimal defect that eventually developed the classic radiological appearance of a carotid web. This case suggests that a focal intimal dissection could be the primum movens, leading to the formation of a web.

\section{Case Report}

A 47-year-old man suffered an episode of transient confusion and perioral tingling. A head CT scan and a CT angiography (CTA) study showed no underlying acute lesions or vascular anomalies (Figs. 1A and 2A). Abnormal- ly low serum blood glucose triggered additional tests that eventually led to the diagnosis of an insulinoma, for which he underwent treatment.

Two years later, the patient presented to our institution with acute-onset left upper motor neuron facial paresis, dysarthria, and left hand paresthesias. His serum glucose level was normal, and head CT scans were negative for acute lesions. Intravenous tissue plasminogen activator was administered, and the patient's neurological examination was normal after 2 hours. Carotid artery ultrasound (US) revealed a proximal raised stump followed by an intimal defect and distal raised intimal flap in the proximal right ICA (Fig. 3A). Review of the dynamic US study did not demonstrate any mobility of the raised intimal flap. A complete stroke workup, including a Holter monitor, thrombophilia screen, and transesophageal echocardiogram, was unremarkable. He was treated with intensive medical therapy (including dual antiplatelet therapy for 3 months and then aspirin monotherapy) and did not suffer recurrent symptoms.

In order to evaluate the evolution of the carotid abnormality, a CTA study obtained 3 months later demonstrated a lesion consistent with a carotid web on the right ICA's posterolateral wall (Figs. 1B and 2B), which persisted at 

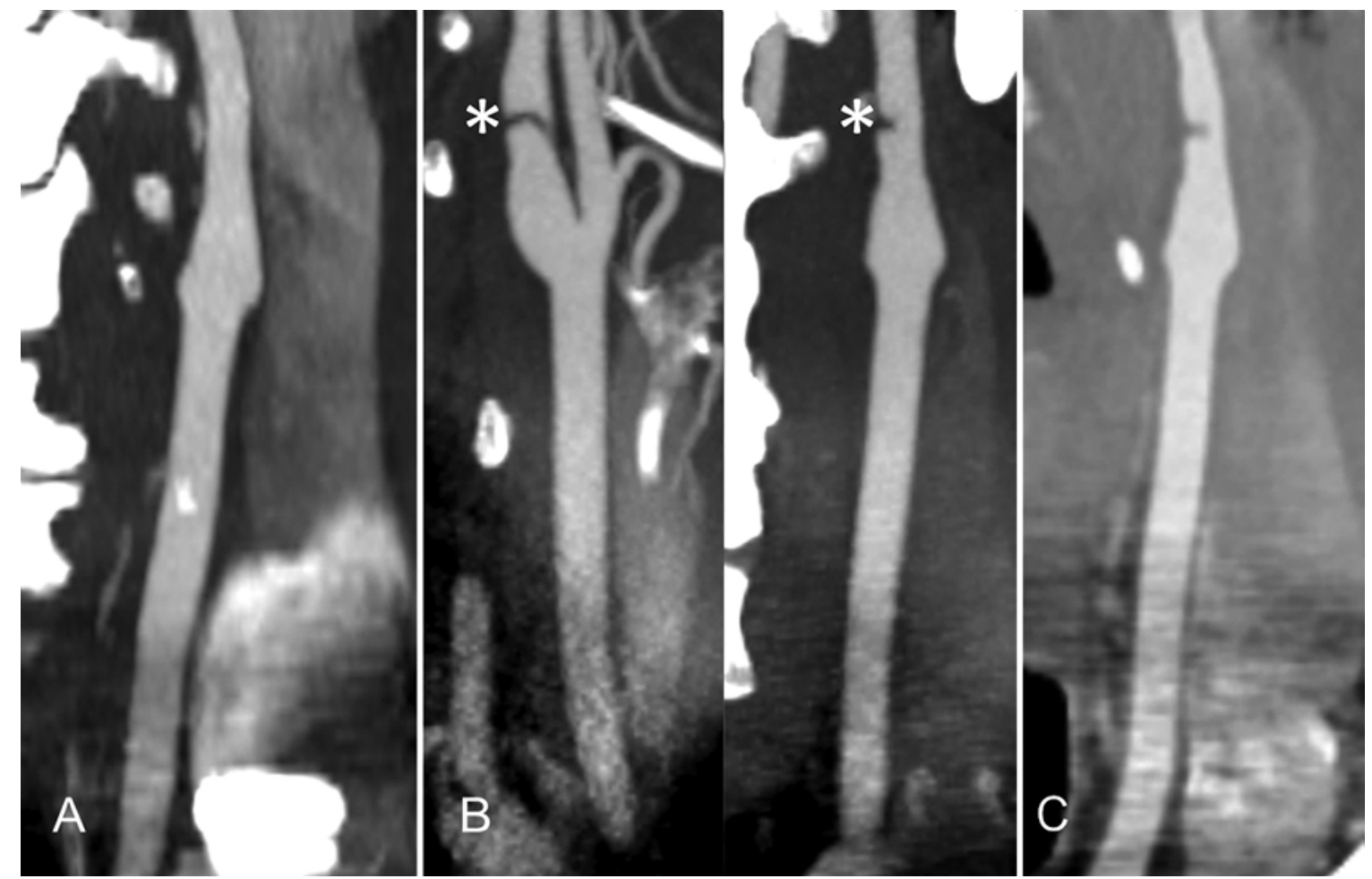

FIG. 1. A: CTA image acquired in 2015 showing a normal aspect of the proximal ICA. B: CTA image obtained 2 years later showing a shelf-like defect (asterisk) in the posterior wall of the proximal right ICA consistent with a carotid web. C: One year later, the carotid web persists.

the 1-year follow-up (Figs. 1C and 2C). A Doppler US repeated 8 months after onset of ischemic symptoms revealed that the proximal raised stump was no longer present, the intimal defect had regenerated, and the distal intimal flap persisted (Fig. 3B).

\section{Discussion}

We document imaging features of the de novo formation of a carotid web on CTA as a result of a focal intimal dissection. Most carotid webs are asymptomatic, and we suggest that a clinically silent dissection could be the cause in those patients with incidentally discovered webs. However, a carotid web is now recognized as a possible and underestimated cause of ischemic stroke in young adults. ${ }^{3,11}$ The etiopathogenesis of a carotid web is controversial, and, until now, its developmental/congenital versus acquired nature has been debated. Histological analysis of surgical specimens shows fibroelastic thickening of the intima without involvement of the tunica media, as is seen in cases of fibromuscular dysplasia., ${ }^{1,2,4,9-13}$

Interestingly, ICA webs are typically located at the posterolateral wall of the vessel. We suggest that the reduced wall shear stress at the posterolateral wall of the carotid bulb, consistently documented in in vitro ${ }^{6}$ and in vivo ${ }^{8,14}$ studies, can lead to greater endothelial vulnerability in this location.? The endothelial reaction to hemodynamic changes at the carotid bulb may create a focal area vulnerable to intimal dissections. In turn, the intimal dissection can evolve morphologically into a "web" and undergo re- active phenomena, leading to a "trapped" area of fibrous tissue within the media. However, carotid webs could be a heterogeneous group of lesions with a similar radiological appearance; as no specimen is available for our case, our hypothesis is mostly theoretical. This hypothesis, supported by the serial imaging findings in our case, explains the characteristic typical location of a carotid web in the posterolateral wall of the proximal ICA and the otherwise unexplained histological findings of fibrous hyperplasia within the intimal layer. Further support to this sequence of events is provided by the histological findings reported by Choi and coworkers. ${ }^{2}$ These authors observed, in 2 of 4 webs they resected at surgery, a dissection splitting the intimal fibrous content.

As carotid webs could be the result and evolution of a prior intimal injury, close follow-up after initial diagnosis should be considered and antiplatelet therapy initiated. In cases presenting with acute symptoms, endovascular or surgical treatment should also be considered. ${ }^{2,5}$

\section{Conclusions}

We documented the de novo formation of a carotid web on serial CTA studies. Based on serial imaging findings in our patient (including high-resolution 2D Doppler US images), we suggest that a focal intimal dissection could be the underlying cause of a carotid web. Lower shear stress at the posterolateral wall of the proximal ICA is hypothesized to be a predisposing factor and to explain the consistent predilection of a carotid web for this location. 
Vercelli et al.

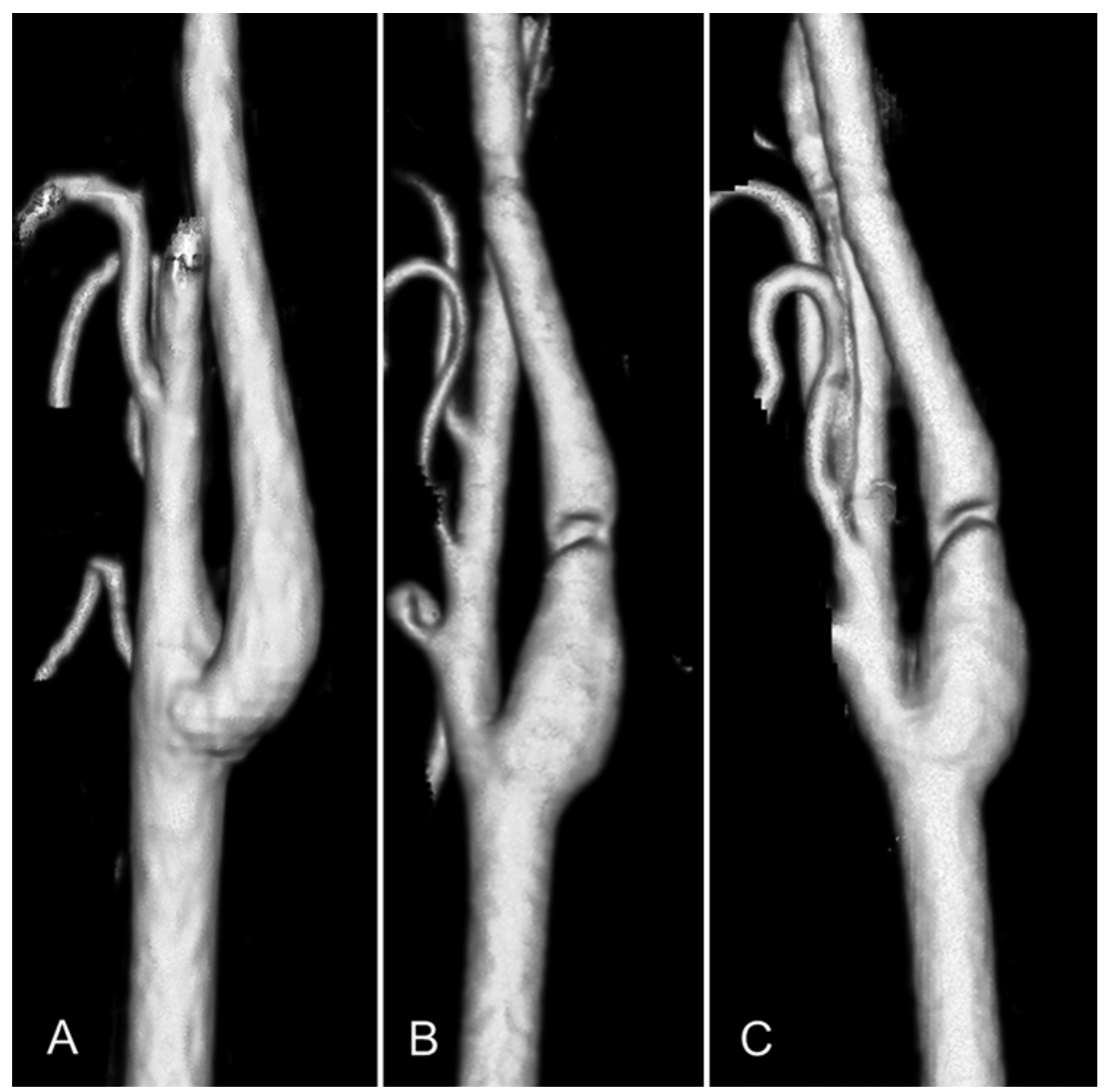

FIG. 2. 3D reconstructions of the right carotid artery bifurcation in 2015 (A), 3 months after onset of ischemia in 2017 (B), and 1 year later $(\mathrm{C})$. There is interval formation of a carotid web (B and $\mathrm{C})$.

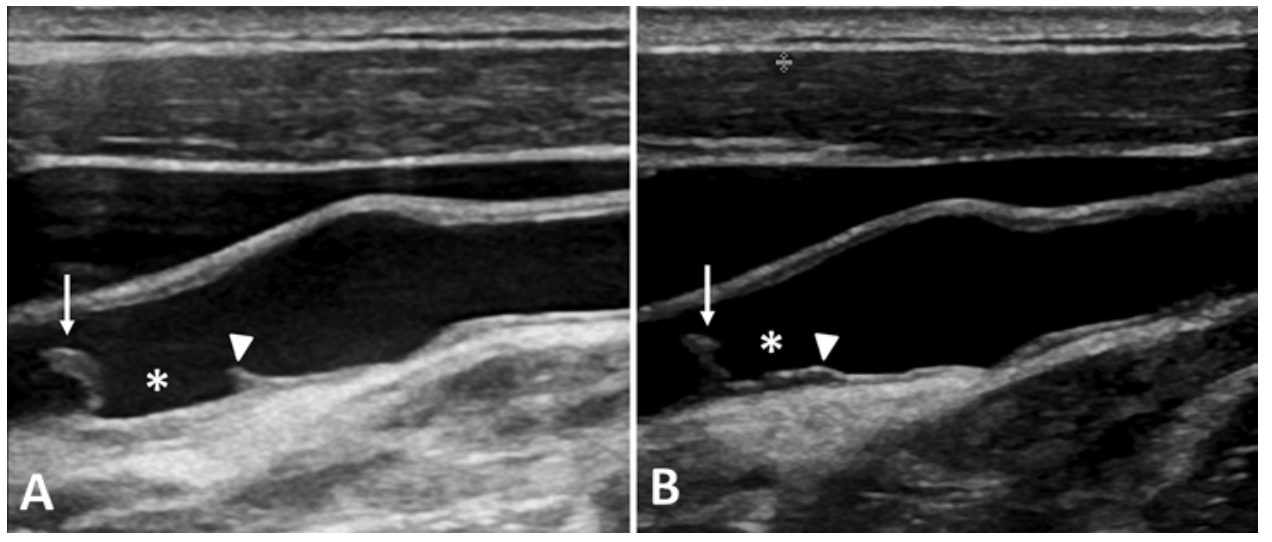

FIG. 3. A: Carotid US image (February 2017) showing a focal defect in the intimal layer (asterisk) and a protruding intimal flap (arrow). There is also a raised "stump" of the intima at the beginning of the intimal defect (arrowhead). B: Follow-up carotid US image (8 months later) demonstrating the re-endothelialization of the intimal defect (asterisk), persistence of the intimal flap (arrow), and remodeling of the raised proximal stump (arrowhead). 


\section{References}

1. Antigüedad-Muñoz J, de la Riva P, Arenaza Choperena G, Muñoz Lopetegi A, Andrés Marín N, Fernández-Eulate G, et al: Internal carotid artery web as the cause of recurrent cryptogenic ischemic stroke. J Stroke Cerebrovasc Dis 27:e86e87, 2018

2. Choi PM, Singh D, Trivedi A, Qazi E, George D, Wong J, et al: Carotid webs and recurrent ischemic strokes in the era of CT angiography. AJNR Am J Neuroradiol 36:2134-2139, 2015

3. Coutinho JM, Derkatch S, Potvin AR, Tomlinson G, Casaubon LK, Silver FL, et al: Carotid artery web and ischemic stroke: a case-control study. Neurology 88:65-69, 2017

4. Gee W, Burton R, Stoney RJ: Atypical fibromuscular hyperplasia involving the carotid artery. Ann Surg 180:136-138, 1974

5. Haussen DC, Grossberg JA, Bouslama M, Pradilla G, Belagaje S, Bianchi N, et al: Carotid web (intimal fibromuscular dysplasia) has high stroke recurrence risk and is amenable to stenting. Stroke 48:3134-3137, 2017

6. Kerber CW, Heilman CB: Flow dynamics in the human carotid artery: I. Preliminary observations using a transparent elastic model. AJNR Am J Neuroradiol 13:173-180, 1992

7. Malek AM, Alper SL, Izumo S: Hemodynamic shear stress and its role in atherosclerosis. JAMA 282:2035-2042, 1999

8. Markl M, Wegent F, Zech T, Bauer S, Strecker C, Schumacher M, et al: In vivo wall shear stress distribution in the carotid artery: effect of bifurcation geometry, internal carotid artery stenosis, and recanalization therapy. Circ Cardiovasc Imaging 3:647-655, 2010

9. Morgenlander JC, Goldstein LB: Recurrent transient ischemic attacks and stroke in association with an internal carotid artery web. Stroke 22:94-98, 1991

10. Phair J, Trestman EB, Yean C, Lipsitz EC: Endarterectomy for a symptomatic carotid web. Vascular 25:553-556, 2017
11. Sajedi PI, Gonzalez JN, Cronin CA, Kouo T, Steven A, Zhuo $\mathrm{J}$, et al: Carotid bulb webs as a cause of "cryptogenic" ischemic stroke. AJNR Am J Neuroradiol 38:1399-1404, 2017

12. Watanabe S, Tanaka K, Nakayama T, Kaneko M: [Fibromuscular dysplasia at the internal carotid origin: a case of carotid web.] No Shinkei Geka 21:449-452, 1993 (Jpn)

13. Wirth FP, Miller WA, Russell AP: Atypical fibromuscular hyperplasia. Report of two cases. J Neurosurg 54:685-689, 1981

14. Xue Y, Gao P, Lin Y, Dai C: Preliminary study of hemodynamics in human carotid bifurcation by computational fluid dynamics combined with magnetic resonance angiography. Acta Radiol 48:788-797, 2007

\section{Disclosures}

The authors report no conflict of interest concerning the materials or methods used in this study or the findings specified in this paper.

\section{Author Contributions}

Conception and design: Lanzino, Vercelli. Acquisition of data: Vercelli. Analysis and interpretation of data: Vercelli, Campeau. Drafting the article: Vercelli. Reviewed submitted version of manuscript: Campeau, Macedo, Dawson. Approved the final version of the manuscript on behalf of all authors: Lanzino. Study supervision: Lanzino.

\section{Correspondence}

Giuseppe Lanzino: Mayo Clinic, Rochester, MN. lanzino. giuseppe@mayo.edu. 